Pacific Journal of Mathematic 


\title{
AN ELEMENTARY PROOF OF THE LIFTING THEOREM
}

\section{TIM TRAYNOR}

\begin{abstract}
An elementary proof is given of the lifting theorem for a complete totally finite measure space, which does not use the martingale theorem or Vitali differentiation.
\end{abstract}

Introduction. In this paper we give a proof of the lifting theorem for a complete totally finite measure space, which involves only elementary properties of measure. The complicated isomorphism theorem of Maharam's original proof [4] is avoided. On the other hand, we do not use the concepts of martingale or of Vitali differentiation ([1] [2] [3] [5]). In fact, the entire construction takes place in the $\sigma$-field of measurable sets, without passing to the algebra of essentially bounded measurable functions. We feel this makes it easier to see what is involved.

Throughout what follows:

$(S, \mathscr{M}, \mu)$ is a complete measure space with $\mu(S)<\infty$;

$\mathscr{N}=\{A \in \mathscr{C l}: \mu(A)=0\}$;

$N$ is the set of nonnegative integers;

For subsets $A, B$ of $S$,

$$
\begin{aligned}
A B & =A \cap B ; \\
A \backslash B & =\{s \in A: s \in B\} ; \\
A^{c} & =S \backslash A ; \\
A \Delta B & =A B^{c} \cup B A^{c} ; \\
A & \doteq B \text { iff } A, B \in \mathscr{C l} \text { and } \mu(A \Delta B)=0 .
\end{aligned}
$$

For a family $\mathscr{K}$ of subsets of $S$,

$$
\bigcup \mathscr{L}=\bigcup_{E \in \mathscr{K}} E .
$$

1. Definitions. For any field $\mathscr{A} \subset \mathscr{A}$,

(1) $d$ is a (lower) density on $\mathscr{A}$ iff $d$ is a mapping on $\mathscr{A}$ to $\mathscr{A}$ such that, for $A, B$ in $\mathscr{A}$,

(i ) $d(A) \doteq A$;

(ii) $A \doteq B$ implies $d(A)=d(B)$;

(iii) $d(\varnothing)=\varnothing, d(S)=S$;

(iv) $d(A B)=d(A) d(B)$.

(2) $l$ is a lifting on $\mathscr{A}$ iff $l$ is a density on $\mathscr{A}$ such that

(v) $l\left(A^{c}\right)=l(A)^{c}$, for $A$ in $\mathscr{A}$.

For a detailed study of liftings and their applications, we refer 
to A. and C. Ionescu Tulcea [3].

2. Remarks. Let $l$ be a lifting on the $\sigma$-field $\mathscr{A} \subset \mathscr{C}$ and $\mathscr{F}=l[\mathscr{A}]$. Then:

(1) $\mathscr{F}$ is a field in $S$.

(2) $\mathscr{F} \subset\{E \in \mathscr{A}: 0<\mu(E)<\mu(S)\} \cup\{\varnothing, S\}$.

(3) If, for each $n$ in $N, E_{n} \in \mathscr{F}$, and $A=\bigcup_{n} E_{n}$, then $l(A) \supset A$. (Indeed, for each $n, E_{n} \backslash l(A) \subset A \backslash l(A) \doteq \varnothing$, so $E_{n} \mid l(A)=\varnothing$, by (2).)

3. Theorem. If $d$ is a density on a field $\mathscr{A}$ with $\mathscr{N} \subset \mathscr{A} \subset \mathscr{A}$, then there exists a lifting $l$ on $\mathscr{A}$, with

$$
d(A) \subset l(A) \subset d\left(A^{c}\right)^{c}, \quad \text { for } A \text { in } \mathscr{A} .
$$

Proof. For each filterbase $\mathscr{\mathscr { B }} \subset \mathscr{A}$, let $\widehat{\mathscr{B}}$ denote an ultrafilter containing $\mathscr{B}$. We recall that for subsets $A, B$ of $S$,

(a) $A \in \widehat{\mathscr{B}}$ iff $A^{c} \notin \widehat{\mathscr{B}}$, and

(b) $A \cap B \in \widehat{\mathscr{B}}$ iff $A \in \widehat{\mathscr{B}}$ and $B \in \hat{\mathscr{B}}$.

For each $s$ in $S$, let

$$
\mathscr{F}(s)=\{A \in \mathscr{A}: s \in d(A)\} \text {. }
$$

Since $d$ is a density, $\mathscr{F}(s)$ is a filterbase. Put

$$
l(A)=\{s \in S: A \in \hat{\mathscr{F}}(s)\}, \quad \text { for } A \text { in } \mathscr{A} .
$$

By the properties (a), (b) of an ultrafilter, for $A, B$ in $\mathscr{A}$, we have (v) $l\left(A^{c}\right)=l(A)^{c}$ and (iv) $l(A B)=l(A) l(B)$. Moreover, if $s \in d(A)$, then $A \in \mathscr{F}(s) \subset \tilde{\mathscr{F}}(s)$, so that $s \in l(A)$. Hence, $d(A) \subset l(A)$. Similarly $d\left(A^{c}\right) \subset l\left(A^{c}\right)$. Using (v) we find that $\left(^{*}\right)$ holds. Since $d(A) \doteq A \doteq d\left(A^{c}\right)^{c}$, we have (i) $l(A) \doteq A$. If $N \doteq \varnothing$, then $d(N)=d(\varnothing)=\varnothing$ and $d\left(N^{c}\right)=$ $d(S)=S$, so that, by $\left(^{*}\right), l(N)=\varnothing$. Hence, (iii) $l(\varnothing)=\varnothing, l(S)=S$ and (ii) if $A \doteq B$, then $l(A) \Delta l(B)=l(A \Delta B)=\varnothing$, so that $l(A)=l(B)$. This completes the proof.

The proof of the following theorem usually uses martingales or Vitali differentiation. We use neither. However, the reader familiar with Sion [5] will recognize the connection with his method. (See Remark 7 below.)

4. THeorem. Suppose that, for each $n$ in $N$, $\mathscr{A}_{n}$ is a $\sigma$-field with $\mathscr{N} \subset \mathscr{A}_{n} \subset \mathscr{A}_{n+1} \subset \mathscr{A}$ and $l_{n}$ is a lifting on $\mathscr{A}_{n}$ with $l_{n}=l_{n+1} \mid \mathscr{A}_{n}$. Put $\mathscr{A}=\sigma$-field $\left(\bigcup_{n} \mathscr{A}_{n}\right)$. Then there is a lifting $l$ on $\mathscr{A}$ with $l_{n}=l \mid \mathscr{A}_{n}$, for each $n$ in $N$. 
Proof. The result will follow immediately from Theorem 3 if we can construct a density $d$ on $\mathscr{A}$ with $d(A)=l_{n}(A)$ for $A$ in $\mathscr{A}_{n}$. To this end, for each $k$ in $N$, let $\mathscr{F}_{k}$ denote $l_{k}\left[\mathscr{A}_{k}\right]$. For each $A$ in $\mathscr{A}, k$ in $N$, and $r<1$, put

$$
\begin{aligned}
\mathscr{D}(A ; k, r) & =\left\{E \in \mathscr{F}_{k}: \mu(A F) \geqq r \mu(F), \text { whenever } E \supset F \in \mathscr{F}_{k}\right\}, \\
d(A ; k, r) & =\bigcup \mathscr{D}(A ; k, r), \text { and } \\
d(A) & =\bigcap_{r<1} \bigcup_{n \in N} \bigcap_{k \geqq n} d(A ; k, r) .
\end{aligned}
$$

We will show that $d$ is a suitable density function on $\mathscr{A}$.

For fixed $A, r$, and $k$, let $\mathscr{K}$ be a maximal disjoint subfamily of $\mathscr{D}(A ; k, r)$. Then $\mathscr{K}$ is countable. Put $B=l_{k}(\cup \mathscr{K})$. Clearly, $B \in \mathscr{D}(A ; k, r)$. Moreover, if $E \in \mathscr{D}(A ; k, r), E \backslash B=\varnothing$, by Remark $2(3)$ and the maximality of $\mathscr{K}$. This shows that $d(A ; k, r)=B$ is the largest element of $\mathscr{D}(A ; k, r)$. In particular, $d(A ; k, r) \in \mathscr{F}_{k} \subset \mathscr{A}$. If $r<s<1$, we have $d(A ; k, r) \supset d(A ; k, s)$, so we need only consider rational $r$. Since $\mathscr{A}$ is a $\sigma$-field, we conclude that $d(A) \in \mathscr{A}$.

There is no difficulty in showing that $A \doteq B \in \mathscr{A}$ implies $d(A)=$ $d(B)$, or that $d(A)=l_{n}(A)$, for $A$ in $\mathscr{A}_{n}$. In particular, $d(\varnothing)=\varnothing$ and $d(S)=S$. We have left to check conditions (i) and (iv) of the definition of a density.

To check condition (iv), let $A, B \in \mathscr{A}, k \in N, r<1$. For each $F$ in $\mathscr{F}_{k}$ contained in $d(A ; k,(r+1) / 2) \cap d(B ; k,(r+1) / 2)$, we have

$$
\begin{aligned}
\mu\left(A B F^{\prime}\right) & =\mu(A F)+\mu(B F)-\mu((A \cup B) F) \\
& \geqq((r+1) / 2) \mu(F)+((r+1) / 2) \mu(F)-\mu(F) \\
& =r \mu(F) .
\end{aligned}
$$

Hence, $d(A ; k,(r+1) / 2) \cap d(B ; k,(r+1) / 2) \subset d(A B ; k, r)$. By direct computation, this yields $d(A) d(B) \subset d(A B)$. On the other hand, for each $k$ and $r, d(A B ; k, r) \subset d(A ; k, r) \cap d(B ; k, r)$, so that $d(A B) \subset$ $d(A) d(B)$, establishing (iv).

To verify condition (i), let $A \in \mathscr{A}$ and put

$$
d^{\prime}(A)=\bigcup_{0<r<1} \bigcap_{n \in N} \bigcup_{k \geqq n} d(A ; k, r) .
$$

We will show that

(a) $d^{\prime}(A) A^{c} \doteq \varnothing$,

(b) $A d^{\prime}\left(A^{c}\right) \doteq \varnothing$, and

(c) $d^{\prime}\left(A^{c}\right) \supset d(A)^{c}, d^{\prime}(A) \supset d(A)$,

from which we get

$$
d(A) \Delta A=d(A) A^{c} \cup A d(A)^{c} \subset d^{\prime}(A) A^{c} \cup A d^{\prime}\left(A^{c}\right) \doteq \varnothing,
$$

as required. 
Fix $r$ in $(0,1)$ and write $D_{k}=d(A ; k, r)$, for $k$ in $N$. Since $D_{k} \in \mathscr{D}(A ; k, r)$, we have for each $B$ in $\mathscr{A}_{k}$,

$$
\mu\left(A B D_{k}\right)=\mu\left(A l_{k}(B) D_{k}\right) \geqq r \mu\left(l_{k}(B) D_{k}\right)=r \mu\left(B D_{k}\right) .
$$

Suppose $B \in \bigcup_{n} \mathscr{A}_{n}$. Then there exists an $n$ in $N$ such that $B \in \mathscr{A}_{n}$. For $m \geqq n, \mathscr{A}_{m} \supset \mathscr{A}_{n}$, and putting $C_{m}=B D_{m} \mid \bigcup_{n \leqq k<m} D_{k}$, we have

$$
\begin{aligned}
\mu\left(A B \bigcup_{k \geqq n} D_{k}\right) & =\sum_{m \geqq n} \mu\left(A C_{m}\right) \\
& \geqq \sum_{m \geqq n} r \mu\left(C_{m}\right) \\
& =r \mu\left(B \bigcup_{k \geqq n} D_{k}\right) .
\end{aligned}
$$

Taking intersections over $n$ we have

$$
\mu\left(A B \bigcap_{n} \bigcup_{k \geqq n} D_{k}\right) \geqq \operatorname{re}\left(B \bigcap_{n} \bigcup_{k \geqq n} D_{k}\right) .
$$

By considering monotone sequences of such $B$ we see that this holds for all $B$ in $\mathscr{A}$, the $\sigma$-field generated by the field $\mathrm{U}_{n} \mathscr{A}_{n}$. In particular, putting $B=A^{c}$ we have $0 \geqq r \mu\left(A^{c} \bigcap_{n} \bigcup_{k \geqq n} D_{k}\right)$. But $r>0$, so $\mu\left(A^{c} \bigcap_{n} \bigcup_{k \geqq n} D_{k}\right)=0$. Taking the union over rational $r$ in $(0,1)$ we have $A^{c} d^{\prime}(A) \doteq \varnothing$. This proves (a). Replacing $A$ by $A^{c}$ we have (b).

To prove (c) we let $k \in N, 0<r<1$ and show

$$
d(A ; k, r)^{c} \subset d\left(A^{c} ; k, 1-r\right) .
$$

To this end suppose $\varnothing \neq E \in \mathscr{F}_{k}$ and $E \subset d(A ; k, r)^{c}$. Then $E \notin$ $\mathscr{D}(A ; k, r)$, so there exists $F$ in $\mathscr{F}_{k}$ contained in $E$ with $\mu(A F)<r \mu(F)$. Let $\mathscr{K}$ be a maximal disjoint collection of such $F$. By Remark 2(3) and maximality of $\mathscr{K}$ we have $E \backslash l_{k}(\mathrm{U} \mathscr{K})=\varnothing$, so $E=l_{k}(\mathrm{U} \mathscr{K})$. Moreover, $\mu(A E)=\sum_{F \in \mathscr{K}} \mu(A F) \leqq \sum_{F \in \mathscr{K}} r \mu(F)=r \mu(E)$. In other words, $\quad \mu\left(A^{c} E\right) \geqq(1-r) \mu(E)$. This shows that $d(A ; k, r)^{c} \in$ $\mathscr{D}\left(A^{c} ; k, 1-r\right)$, so $d(A ; k, r)^{c} \subset d\left(A^{c} ; k, 1-r\right)$. Hence,

$$
\begin{aligned}
d(A)^{c} & =\bigcup_{r \in(0,1)} \bigcap_{n} \bigcup_{k \geqq n} d(A ; k, r)^{c} \\
& \subset \underset{r \in(0,1)}{ } \bigcap_{n} \bigcup_{k \geqq n} d\left(A^{c} ; k, 1-r\right) \\
& =d^{\prime}\left(A^{c}\right) .
\end{aligned}
$$

Since it is clear that $d(A) \subset d^{\prime}(A)$, this proves (c) and completes the proof of the theorem.

To prove the lifting theorem, we need one more lemma, due to A. and C. Ionescu Tulcea [2]. For completeness, we include a proof here.

5. Lemma. Let $\mathscr{A}$ be a $\sigma$-field with $\mathscr{N} \subset \mathscr{A} \subset \mathscr{H}, l$ a lifting 
on $\mathscr{A}$. If $A \in \mathscr{M} \backslash \mathscr{A}$ and $\mathscr{A}^{\prime}=$ field $(\mathscr{A} \cup\{A\})$, then there exists a lifting on $\mathscr{A}^{\prime}$ extending $l$.

Proof. Let $\mathscr{F}=l[\mathscr{A}], \mathscr{E}=\left\{E \in \mathscr{F}: \mu\left(E A^{c}\right)=0\right\}$. Let $\mathscr{K}$ be a maximal disjoint subfamily of $\mathscr{E}$ and let $A_{1}=l(\bigcup \mathscr{K})$. Then $A_{1} \in \mathscr{E}$ and, by maximality of $\mathscr{K}$ and Remark 2(3), $E \backslash A_{1}=\varnothing$, for all $E$ in $\mathscr{E}$, so that $A_{1}$ is the largest element of $\mathscr{E}$. Similarly, let $A_{2}$ be the largest $E$ in $\mathscr{F}$ with $\mu(E A)=0$. Put $\bar{A}=\left(A \cup A_{1}\right) \backslash A_{2}$. Then $\bar{A} \doteq A$. (Indeed, $\bar{A} \triangle A \subset A_{1} A^{c} \cup A_{2}^{c} A \doteq \varnothing$.) Thus, $\mathscr{A}^{\prime}=$ field $(\mathscr{A} \cup\{\bar{A}\})\left(=\left\{\left(C \bar{A} \cup D \bar{A}^{c}: C, D \in \mathscr{A}\right\}\right)\right.$. For $E, F$ in $\mathscr{F}$,

(a) $E \bar{A} \doteq F \bar{A}$ implies $E \bar{A}=F \bar{A}$, and

(b) $E \bar{A}^{c} \doteq F \bar{A}^{c}$ implies $E \bar{A}^{c}=F \bar{A}^{c}$.

Indeed, $E \bar{A} \doteq F \bar{A}$ implies $\mu((E \Delta F) A)=\mu((E \Delta F) \bar{A})=0$, so that, by definition of $A_{2}, E \Delta F \subset A_{2} \subset \bar{A}^{c}$. Thus, $(E \Delta F) \bar{A}=\varnothing$, so $E \bar{A}=F \bar{A}$. The proof of (b) is similar.

Now define $l^{\prime}$ on $\mathscr{C}^{\prime}$ by

$$
l^{\prime}\left(C \bar{A} \cup D \bar{A}^{c}\right)=l(C) \bar{A} \cup l(D) \bar{A}^{c}, \quad \text { for } C, D \text { in } \mathscr{A} .
$$

Using (a) and (b) we see that $l^{\prime}$ is well-defined and that for $M_{1}, M_{2}$ in $\mathscr{A}^{\prime}, M_{1} \doteq M_{2}$ implies $l^{\prime}\left(M_{1}\right)=l^{\prime}\left(M_{2}\right)$. The other properties of a lifting are easily verified. Moreover, for $C$ in $\mathscr{A}, l^{\prime}(C)=l(C) \bar{A} \cup l(C) \bar{A}^{c}=l(C)$, so $l^{\prime}$ extends $l$.

We can now prove the lifting theorem:

6. Theorem. Let $(S, \mathscr{M}, \mu)$ be a complete measure space with $\mu(S)<\infty$. Then, there exists a lifting on $\mathscr{M}$.

Proof. Let $\mathscr{H}$ be the set of pairs $(\mathscr{A}, l)$ where $\mathscr{A}$ is a $\sigma$-field with $\mathscr{N} \subset \mathscr{A} \subset \mathscr{M}$ and $l$ is a lifting on $\mathscr{A}$, with the ordering: $(\mathscr{A}, l) \leqq$ $\left(\mathscr{A}^{\prime}, l^{\prime}\right)$ iff $\mathscr{A} \subset \mathscr{A}^{\prime}$ and $l=l^{\prime} \mid \mathscr{A}$. We show that $\mathscr{H}$ has a maximal element. Indeed, suppose $\mathscr{H}^{\prime}=\left\{\left(\mathscr{A}_{i}, l_{i}\right): i \in I\right\}$ is a totally ordered subfamily of $\mathscr{H}$. We distinguish two cases:

(a) If $\mathscr{H}^{\prime}$ has no countable cofinal subfamily, put $\mathscr{A}=\mathrm{U}_{i \in I} \mathscr{A}_{i}$ and $l(A)=l_{i}(A)$, for $A$ in $\mathscr{A}_{i}, i$ in $I$. Then $(\mathscr{A}, l)$ is an upper bound for $\mathscr{H}^{\prime}$ in $\mathscr{H}$.

(b) If $\mathscr{H}^{\prime}$ has a countable cofinal subfamily $\mathscr{H}^{\prime \prime}=\left\{\left(\mathscr{A}_{i_{n}}, l_{i_{n}}\right)\right.$ : $n \in N\}$, then by Theorem 4, $\mathscr{H}^{\prime \prime}$ (and hence $\mathscr{H}^{\prime}$ ) has an upper bound in $\mathscr{H}$. By Zorn's lemma, we conclude that $\mathscr{H}$ has a maximal element, $(\mathscr{A}, l)$.

By Lemma 3, and maximality, $\mathscr{A}=\mathscr{A}$, and the theorem is proved.

7. REMARKS.

(1) To see the relationship of our method to that of Sion [5], 
for each $k$ in $N$ and $s$ in $S$, let $\hat{\mathscr{F}}_{k}(s)=\left\{F \in \mathscr{F}_{k}: s \in F\right\}$, directed downward by inclusion. Then,

$$
d(A ; k, r)=l_{k}\left(\left\{s \in S: \underline{\lim }_{F \in \hat{\sim}_{k}(s)} \frac{\mu(A F)}{\mu(F)} \geqq r\right\}\right) .
$$

(One inclusion is obvious, the other follows from Sion's Theorem 2'.)

(2) As several authors have pointed out (see, for example, Sion [5], and for more references, Sion [6]), liftings provide very special Vitali differentiation system, even when no others are available. (If $l$ is a lifting on $\mathscr{C}$, such a system is obtained by assigning to each $s$ in $S,\{F: s \in F \in l[\mathscr{C}]\}$, directed downward by inclusion.) Apart from our desire for an elementary proof, this was our main motivation in looking for a construction of a lifting without using differentiation concepts.

(3) Added in proof. S. Graf [On the existence of strong: liftings in second countable topological spaces, (to appear)] has noticed that one may change the word "lifting" to "density" in the statement of Theorem 4. The proof is essentially contained in our proof. Graf has independently obtained a proof of this result (using RadonNikodým derivatives).

\section{REFERENCES}

1. G. Hansel, Théorème de relèvement et mesures bivalent, Ann. Inst. Poincaré 8 (1972), 395-401.

2. A. and C. Ionescu Tulcea, On the lifting property I, J. Math. Annal. Appl., 3 (1961), 537-546.

3. - Topics in the Theory of Lifting, Springer-Verlag, 1969.

4. D. Maharam, On a theorem of Von Neuman, Proc. Amer. Math. Soc., 9 (1958), 987-994.

5. M. Sion, A proof of the lifting theorem, Univ. of British Columbia, 1970.

6. - A Theory of Semigroup-valued Measures, Univ. of British Columbia 197172.

Received August 1, 1973 and in revised form October 3, 1973. This work was supported by the Summer Research Institute of the Canadian Mathematical Congress.

UNIVERSITY OF WINDSOT 


\section{PACIFIC JOURNAL OF MATHEMATICS}

\section{EDITORS}

RICHARD ARENS (Managing Editor)

University of California

Los Angeles, California 90024

R. A. Beaumont

University of Washington

Seattle, Washington 98105
J. DugundJI

Department of Mathematics

University of Southern California

Los Angeles, California 90007

D. Gilbarg and J. Milgram

Stanford University

Stanford, California 94305

\section{ASSOCIATE EDITORS}

E. F. BECKENBACH

B. H. NeUMANN

F. WOLF

K. YosHIDA

\section{SUPPORTING INSTITUTIONS}

UNIVERSITY OF BRITISH COLUMBIA

CALIFORNIA INSTITUTE OF TECHNOLOGY

UNIVERSITY OF CALIFORNIA

MONTANA STATE UNIVERSITY

UNIVERSITY OF NEVADA

NEW MEXICO STATE UNIVERSITY

OREGON STATE UNIVERSITY

UNIVERSITY OF OREGON

OSAKA UNIVERSITY
UNIVERSITY OF SOUTHERN CALIFORNIA

STANFORD UNIVERSITY

UNIVERSITY OF TOKYO

UNIVERSITY OF UTAH

WASHINGTON STATE UNIVERSITY

UNIVERSITY OF WASHINGTON

$\stackrel{*}{*} \stackrel{*}{*} \stackrel{*}{*}$ AMERICAN MATHEMATICAL SOCIETY




\section{Pacific Journal of Mathematics}

\section{Vol. 53, No. $1 \quad$ March, 1974}

Martin Bartelt, Strongly unique best approximates to a function on a set, and a finite

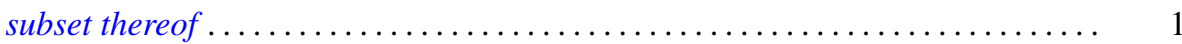

S. J. Bernau, Theorems of Korovkin type for $L_{p}$-spaces $\ldots \ldots \ldots \ldots \ldots \ldots \ldots \ldots \ldots$

S. J. Bernau and Howard E. Lacey, The range of a contractive projection on an

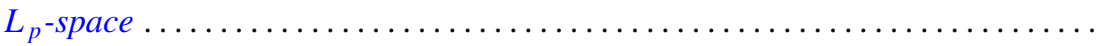

Marilyn Breen, Decomposition theorems for 3-convex subsets of the plane ......... Ronald Elroy Bruck, Jr., A common fixed point theorem for a commuting family of

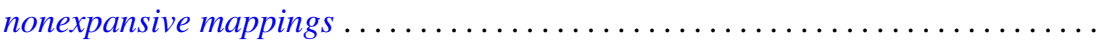

Aiden A. Bruen and J. C. Fisher, Blocking sets and complete $k$-arcs . . . . . . . 73

R. Creighton Buck, Approximation properties of vector valued functions . ......... 85

Mary Rodriguez Embry and Marvin Rosenblum, Spectra, tensor products, and

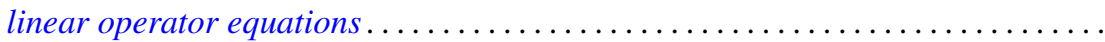

Edward William Formanek, Maximal quotient rings of group rings . . . . . . . . . 109

Barry J. Gardner, Some aspects of T-nilpotence . . . . . . . . . . . . . . . 117

Juan A. Gatica and William A. Kirk, A fixed point theorem for $k$-set-contractions

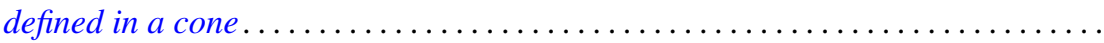

Kenneth R. Goodearl, Localization and splitting in hereditary noetherian prime

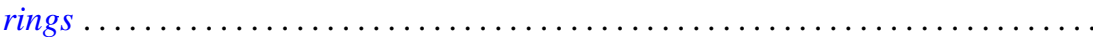

James Victor Herod, Generators for evolution systems with quasi continuous

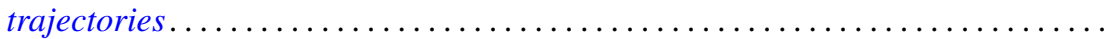

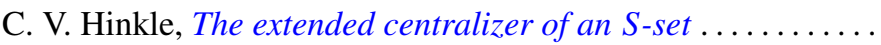

I. Martin (Irving) Isaacs, Lifting Brauer characters of p-solvable groups . . .

Bruce R. Johnson, Generalized Lerch zeta function ...........

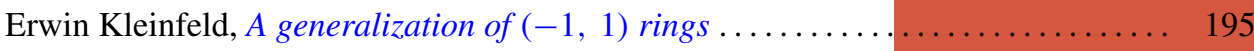

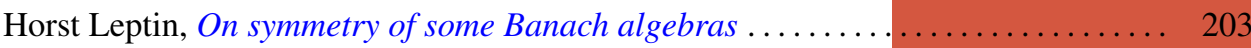

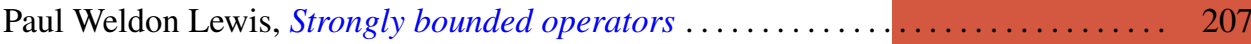

Arthur Larry Lieberman, Spectral distribution of the sum of self-adjoint

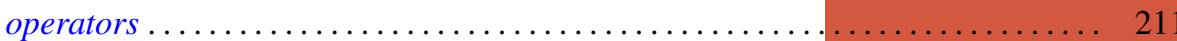

I. J. Maddox and Michael A. L. Willey, Continuous operators on paranormed spaces and matrix transformations

James Dolan Reid, On rings on groups ........................... 229

Richard Miles Schori and James Edward West, Hyperspaces of graphs are Hilbert cubes.

William H. Specht, A factorization theorem for p-constrained groups ...

Robert L Thele, Iterative techniques for approximation of fixed points of certain nonlinear mappings in Banach spaces ...............

Tim Eden Traynor, An elementary proof of the lifting theorem

Charles Irvin Vinsonhaler and William Jennings Wickless, Completely decomposable groups which admit only nilpotent multiplications .

Raymond O’Neil Wells, Jr, Comparison of de Rham and Dolbeault cohomology for

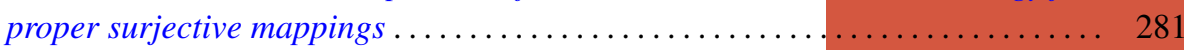

David Lee Wright, The non-minimality of induced central representations . . . . . 301 